\title{
Phyllodes tumor in pregnancy: A Case Report
}

\author{
Patil P. ${ }^{1}$, Rajpal T. ${ }^{2}$, Pandya B. ${ }^{3}$, Brahmachari S. ${ }^{4}$
}

${ }^{1}$ Dr. Pooja Patil, Professor, ${ }^{2}$ Dr. Tanya Rajpal, Resident, Department of Obstetrics and Gynecology, L.N.M.C. \& JKHMRC, Bhopal, ${ }^{3}$ Dr. Bharati Pandya, Additional Professor, ${ }^{4}$ Dr. Swagata Brahmachari, Associate Professor, Department of Surgery, AIIMS,Bhopal, India.

Correspondence Author: Dr. Tanya Rajpal, Resident, Department of Obstetrics and Gynecology, L.N.M.C. \& JKHMRC, Bhopal, India. E-mail: trajpal5@gmail.com

\begin{abstract}
Phyllodes tumor accounts for less than $1 \%$ of all breast neoplasms. These tumors are fast growing masses arising from the periductal stromal cells of the breast. We report a case of phyllodes in a 25 year old primigravida who presented to us at 18 weeks of pregnancy with mass of right breast which appeared 15 days back.

She gave history of some mass in right breast for which she was operated 1 year back for which no details or papers were available. On examination there was a tumor in Right breast of about 20x15x11 cm size.ANC examination showed uterus of 18 wks size, FHS 140/min, regular. Patient underwent FNAC and biopsy from the mass which was suggestive of malignant Phyllodes tumor.

Patient was prepared for mastectomy and along with surgical team right mastectomy with right axillary lymph node dissection was done. Post-operative period was uneventful. Histopathological examination showed increased cellularity and nuclear atypia suggestive of malignant phyllodes tumour and lymph nodes were negative. We concluded that accurate preoperative pathological diagnosis is very important for management of phyllodes tumor, and allows correct surgical planning and avoidance of reoperation.
\end{abstract}

Keywords: Phyllodes tumor, Pregnancy, breast tumour

\section{Introduction}

Phyllodes tumor are rare, accounting for less than $1 \%$ of all breast neoplasms [1]. These tumors are fast growing tumor masses arising from the stromal cells around the ducts of the breast. Its incidence is more common in the $4^{\text {th }}-5^{\text {th }}$ decade of life prior to menopause. These tumors have rapid growth and the associated symptoms can mimic other types of breast carcinoma, particularly if the mass ulcerates and bleeds.

Malignant tumors usually have rhabdomyosarcoma and liposarcoma rather than fibrosarcomas, the number of mitoses is of great help in the diagnosing the malignant subtype [2-4].

Local recurrence rate of phyllodes tumor is high and it has a little tendency to metastasize to distant organs. All forms of phyllodes tumors have malignant potential. Like sarcomas, they can

Manuscript received:20 $0^{\text {th }}$ January 2019

Reviewed:30 $30^{\text {th }}$ anuary 2019

Author Corrected: $7^{\text {th }}$ February 2019

Accepted for Publication: $12^{\text {th }}$ February 2019 metastasise to various organs via blood route, commonly the lungs, bone, and abdominal viscera [3]. The majority of phyllodes tumors are benign $(35 \%$ to $64 \%$ ), while rest of them could be eitherborderline or malignant subtype. Benign tumors have a five-year survival rate of almost $100 \%$ o, $98 \%$ with borderline, and about $88 \%$ with malignant [5]. Phyllodes tumors often present a diagnostic and treatment dilemma.

Primary treatment for phyllodes tumor is surgical.Wide local excision is the treatment of choice and it mainly depends on the tumor size.

Primary treatment of the tumor includes local excision of the tumor mass.However in spite of wide excision, a large number of surgeries yield incomplete excision margins and thus may require revision surgery. 


\section{Case Report}

A 25 year old primigravida presented to us in J.K. Hospital, Bhopal with 5 months of amenorrhoea with mass of right breast which appeared 15 days back. The mass was insidious in onset and increased rapidly to the current size and was non-tender. There was no ulceration or bleeding from the surface of the tumor. Her first trimester was uneventful. She was booked, immunized and was on regular ANC follow-up.

She gave history of some mass in right breast for which she was operated 1 year back. Excision of the mass was done in another hospital for which no details or papers were available. No other significant past medical or surgical history, except that the mass aggressively reoccurred again.

There was no family history of the same illness. Her sleep, appetite, bowel and bladder function were all normal.

On presentation, the patient was alert, conscious and her vital signs were stable. General examination revealed no pallor, icterus, edema or cyanosis. Her cardiovascular and respiratory system examination was within normal limits.

On breast examination, a giant tumor in right breast of about $20 \times 15 \mathrm{~cm}$ size was evident. Per abdomen examination showed uterus of 18 weeks size with a regular fetal heart rate of 140 beats per minute.

Laboratory and Imaging findings- All ANC investigations were normal including ultrasound. Patient underwent FNAC and biopsy from the mass which was suggestive of malignant phyllodes tumor.

Surgery and Post-operative findings- Patient was prepared for mastectomy and along with surgical team right mastectomy with right axillary lymph node dissection was done.

Macroscopic findings- A breast tissue measuring $20 \times 15 \times 11 \mathrm{~cm}$ in dimensions was resected (Figure 1). Skin over the tumor was tan in color. Cut surfacerevealed fleshy multiple nodules of variable size, soft to firm in consistency.

Microscopic Findings: Breast tissue and nodular masses were consistent with fibro adenomatous changes.Stromal dominance was noted and the stromal and glandular tissues were poorly organized. There were areas of hemorrhage, thrombosis, and necrosis. Microscopic examination showed increased mitosis. There was stromal and epithelial proliferation with leaf pattern epithelial component of the tumor growth. Therefore, final histological diagnosis was malignant phyllodes tumor.

Post-operative period was uneventful. Histopathological examination showed increased cellularity and nuclear atypia which was suggestive of malignant phyllodes tumour and lymph nodes were found to be negative.
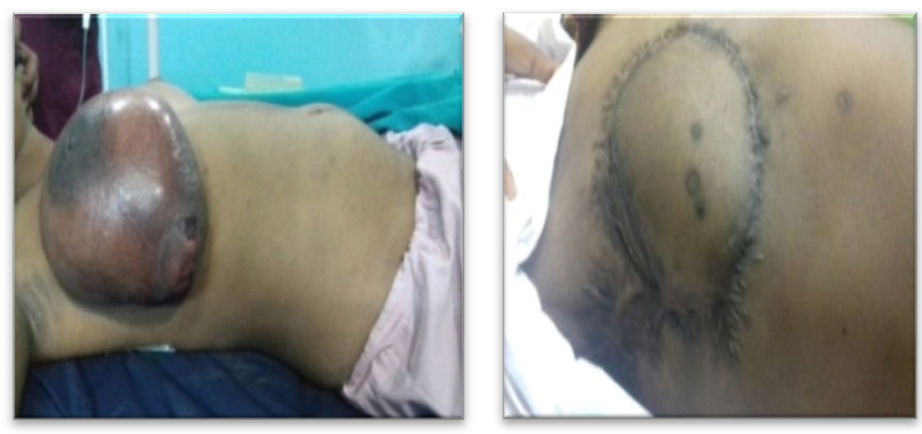

Fig-1: Pre operative image of tumorFig-2: Post operative image of tumor Size 20x15cmafter 6 months of surgical resection

Follow-up and pregnancy outcome- Patient came for regular antenatal check-ups. At term, patient had spontaneous labor pains and delivered vaginally a male child of $2.5 \mathrm{~kg}$ with APGAR score 8/10. Patient came for follow up after 6 months and the baby and mother were both doing well. 


\section{Discussion}

The biggest challenge to the treating physician is that of local recurrence or metastasis of tumor. Phyllodes tumor appears to grow more rapidly during pregnant state. A variety of investigations including mammography, biopsy, USG, MRI have been used for making the correct diagnosis.

Certain hormones play vital role in proliferation in lobular and alveolar tissue of the breast, for example progesterone, estrogen, human chorionic gonadotropin hormone, angiogenic factors and vascular endothelial growth factor during pregnancy.

These tumors grow radially and compress the surrounding breast parenchyma.Thetumor is surrounded by a false capsule and the tumor extends and grows into the rest of the healthy mammary tissue through this capsule [6]. The overlying skin is usually shiny and translucent. Underlying veins are visible on the surface of tumor at its initial presentation.

Phyllodes tumor in most of the cases mimic benign breast condition such as fibroadenoma.This is challenging in making a diagnosis. The tumor differentiates from other benign breast disorders by the increased mitotic activity, cellular atypia, and stromal proliferation. Lungs are the most common metastatic site although very rare; followed by the skeleton, heart and liver [7]. The definitive methods for diagnosing the phyllodes tumor are incisional and excisional biopsies. Core cut biopsy is proven to be a reliable tool for diagnosing these tumors.

Surgery: Primary treatment for phyllodes tumor is surgical.Treatment of choice is wide local excision depending on the size of the tumor. If margins of $1 \mathrm{~cm}$ cannot be attained then simple mastectomy is the next best option. Overall survival rates have been noted to be similar in patients undergoing wide local excision with adequate margins and those undergoing mastectomy [8, 9].Local recurrences are common in phyllodes tumor to avoid future recurrence of the tumor, mastectomy with axillary node dissection would be the appropriate surgical option.

Role of Radiotherapy: Belkacemi et al. reported that radiotherapy was associated with superior local control rate at 10 years, from $59 \%$ to $86 \%$ for both borderline and malignant phyllodes tumors [10].However there is insufficient data regarding patients with metastatic disease being treated with radiotherapy.
Role of Chemotherapy: Adjuvant chemotherapy could possibly be offered to patients with large $(>5 \mathrm{~cm})$, highrisk or recurrent malignant tumors. Turalba et al. showed that doxorubicin and ifosfamide-based chemotherapies have role in women with metastatic phyllodes tumors [11].

Hormonal therapy is however not effective in phyllodes tumor.The 5-year overall-survival rates for benign and malignant phyllodes tumor were reported to be approximately $90 \%$ and $80 \%$, respectively[9].However, lower survival rates for malignant tumors have been noted by some authors [12].

After surgery,patient should be on a regular close follow-up with frequent breast examinations and radiological testing.

Our patient had history of the previous excision and with a large tumor presently, high mitotic rate, poorly organized variably arranged stroma, marked pleomorphism, all in favour of malignant etiology. In spite of all of these findings, she was operated with proper surgical margins and on close follow up till 6 months after delivering the baby, and there was no recurrence.

\section{Conclusion}

Cystosarcoma Phyllodes is a rare tumor of the breast occurring more commonly in the peri-menopausal age group. However, our patient being in the reproductive age group presented with this rare entity with no significant effect on her pregnant state. These tumors have a higher rate of local recurrence.

This patient also presented with a similar pattern of recurrence as she gave a history of previous excision done in the same breast one year back. Accurate pathological diagnosis before planning surgery allows resection of tumor with adequate margins and avoidance of reoperation.

The value of FNAC in the diagnosis of phyllodes tumor remains controversial. Nonetheless, core needle biopsy is found to be having high sensitivity. Surgery is the mainstay of treatment of such tumors. Local recurrence in phyllodes tumors has been associated with inadequate local excision.

Funding: Nil, Conflict of interest: Nil

Permission from IRB: Yes 


\section{Case Report}

\section{References}

1. Murat ÖzgürKılıç, Serdar GökayTerzioğlu, Betül Bozkurt, and GülDağlar. Phyllodes Tumor of the Breast: Analysis of 48 Patients. J Breast Health2016 Oct; 12(4): 158-164. doi: 10.5152/tjbh.2016.3100

2. Kumar T, Patel MD, Bhargavan R, Kumar P, Patel $\mathrm{MH}$, Kothari K, et al. Largest Phyllodes Tumor- Case Report and Brief Review Article. Indian J Surg Oncol. 2011 Jun; 2(2): 141-144. Published online 2011 Jul 15. doi: 10.1007/s13193-011-0077-3.

3. Moffat CJ, Pinder SE, Dixon AR, Elston CW, Blamey RW, Ellis IO. Phyllodestumour of the breast: a clinicopathological review of the thirty-two cases.Histopathology.September 1995;27(3):205-218. doi: 10.1111/j.1365-2559.1995.tb00212.x.

4. Rowell MD, Perry RR, Jeng-Gwang H, Barranco SC. Phyllodes tumors. Am J Surg.March 1993;165(3)376379. doi: 10.1016/S0002-9610(05)80849-9.

5. Ye John Shangming Seven Lakes High School, Katy, TX. Statistical analysis on the behavior and recurrence of breast phyllodes tumors. J Clin Oncol. 2016;34(suppl 3)

6.Adamietz B. Differentiation Between Phyllodes Tumor and Fibroadenoma Using Real-Time Elastography. Ultraschall Med. 2011;32(Suppl 2):E75-9. doi: 10.1055/s-0031-1282024.
7.Abe M, Miyata S, Nishimura S, et al. Malignant transformationof breast fibroadenoma to malignant phyllodes tumor: long-term outcome of 36 malignant phyllodes tumors. Breast Cancer. 2011Oct;18(4):26872. doi: 10.1007/s12282-009-0185-x.

8. Cheng S., Chang Y. Phyllodes tumor of the breast: thechallenge persists. World J. Surg. August 2006;30(8):1414-21. DOI:10.1007/s00268-005-0786-2

9. Macdonald OK, Lee CM, Tward JD, et al. Malignant phyllodes tumor of the female breast: association of primary therapy with cause-specific survival from the Surveillance, Epidemiology, and End Results (SEER) program. Cancer. 2006 Nov 1;107(9):2127-33.

10. Belkacémi Y, Bousquet $\mathrm{G}$, Marsiglia $\mathrm{H}$, et al. Phyllodes tumor of the breast. Int J Radiat Oncol Biol Phys. 2008 Feb 1;70(2):492-500. Epub 2007 Oct 10.

11. Turalba CI, el-Mahdi AM, Ladaga L. Fatal metastatic cystosarcoma phyllodes in an adolescent female: case report and review of treatment approaches. J Surg Oncol. 1986 Nov;33(3):176-81.

12. Suzuki-Uematsu S, Shiraishi K, Ito T, Adachi N, et al. Malignant phyllodes tumor composed almost exclusively of a fibrosarcomatous component: a case report and review of malignant phyllodes tumors with metastases. Breast Cancer. 2010 Jul;17 (3): 21824.doi:10.1007/s12282-009-0099-7. Epub2009Apr 7

\section{How to cite this article?}

Patil P, Rajpal T, Pandya B, Brahmachari S.Phyllodes tumor in pregnancy: A Case Report.Obs Rev:J obstet Gynecol 2019;5(1):30-33.doi:10.17511/joog.2019.i01.06 